“ (C) 2018 IEEE. Personal use of this material is permitted. Permission from IEEE must be obtained for all other uses, in any current or future media, including

reprinting/republishing this material for advertising or promotional purposes, creating new collective works, for resale or redistribution to servers or lists, or reuse of any copyrighted component of this work in other works." 


\title{
A Frequency-Limited Adaptive Controller for Underwater Vehicle-Manipulator Systems Under Large Wave Disturbances
}

\author{
Wenjie Lu and Dikai Liu
}

\begin{abstract}
Standard adaptive control approaches may not be able to sufficiently stabilize underwater vehicle-manipulator systems (UVMSs) when wave disturbances are large, leading to high-frequency oscillations of large amplitude in its dynamic model parameters. Such parameters bring about undesired oscillations in the vehicle body control and state. This paper extends a frequency-limited adaptive control approach to the vehicle body. An auxiliary model is obtained from the approximated model through a low-pass filter and is used to reduce the problematic oscillations. The resultant stable vehicle body is a necessary premise to successfully finding manipulator control for end-effector tracking. In addition, this paper proposes a sufficient condition of the control gains for guaranteed asymptotical stability of the controlled robotic system. Numerical simulations have demonstrated the effectiveness of the presented approach, compared to the standard adaptive control.
\end{abstract}

\section{INTRODUCTION}

The exploitation of hostile underwater environments highly depend on the performance of one or more underwater robots equipped with several manipulators, referred to as Underwater Vehicle-Manipulator Systems (UVMSs) [1]. These systems can be used in a wide range of practical applications, such as shipwreck search and rescue, underwater structure maintenance, and biological sampling. UVMSs of large size and heavy weight have been developed and studied through several projects, such as ALIVE [2], SAUVIM [3],TRIDENT [4],TRITON [5], and PANDORA [6].

Recently, small UVMSs operating near offshore and in shallow water are gaining more attention in applications such as bridge pier cleaning and inspection [7] and benthic habitat mapping [8]. Different than the ones operating in deep sea, these robotic systems may experience wave disturbances of much higher frequency and of much larger amplitude. The wave disturbances impose hydrodynamic effects (e.g., added mass and inertia and damping effects) to the UVMSs. These effects depend heavily on the fluid conditions and UVMS characteristics, such as fluid density and velocities, and UVMS effective area and velocities, etc. [9]. These conditions and thus the hydrodynamic effects vary temporally and spatially and they can rarely be known in practical applications, bringing about challenging control problems of uncertain, time variant, and nonlinear systems.

\footnotetext{
*This work was supported by the Australian Research Council (ARC) Linkage Project (LP150100935), the Roads and Maritime Services of NSW, and the Centre for Autonomous Systems (CAS) at the University of Technology Sydney.

Authors are with CAS at the University of Technology Sydney, wenjie.lu@uts.edu. au
}

To overcome the aforementioned issues, several control techniques have been developed, such as output feedback control [10], sliding mode control [11], and fuzzy logic control [12], etc. Adaptive control is one popular solution and has been studied by many researchers [13], [14], [15], [16]. It approximates the unknown UVMS dynamics online via a parameterized model. This parameterized model is referred to as the approximation model and is used to adapt the control gain to modeling parameters that are unknown. One popular model candidate is derived from Newton-Euler equations of motion of the UVMS using the property of linearity in parameters [17]. Another choice of the model employs a set of basis functions (e.g., sigmoid functions) to form a one-layer neural network. The model derived from Netwon-Euler equations of motion incorporates the knowledge about the UVMS dynamics and is therefore more robust than the the neural network model when observation data available cannot sufficiently cover entire robot state of interest. A non-regressor adaptive control method also is reported in [18].

As presented in [13], [19], the standard adaptive control approach can sufficiently adaptive the system when the hydrodynamic effects vary slowly. However in shallow water and near shore applications, dramatic changes occur rapidly in wave disturbances and hydrodynamic effects. The tracking errors of the vehicle body at instants of these changes, referred to as transient performance, is usually large and of high-frequency oscillation. This is because the adaptation of modeling parameters is proportional to the mismatch between the outputs from the actual vehicle body and the outputs from the approximation model of body dynamics. The large mismatches at transient instants result in an overshooting of parameter adaption, leading to highfrequency oscillations in the error of dynamics modeling. The control calculated from such problematic approximation model makes the vehicle body oscillate.

In this paper, similar to the idea from [20], an auxiliary reference model is adopted to filter out high-frequency oscillations in modeling of the vehicle body dynamics during transient instants. The discrepancy between the approximation model and this auxiliary model is used to counteract the high-frequency oscillations in adapting modeling parameters. Furthermore, a sufficient condition of parameters in control design is presented for stabilizing the robotic system .

According to [17], it usually requires more than 20 parameters to sufficiently model one underwater rigid body and thus it is inefficient to model every module in the UVMS. Besides, the vehicle body controlled by thrusters usually has 
low frequency response than the manipulator controlled by motors. Also in practice, each joint of the manipulator is effectively and independently controlled via PID approaches given the desired joint velocity and/or positions. Therefore, similar to [21], the control scheme here considers the vehicle body system separately from the manipulator and employs two controllers with different bandwidths. In this paper, the control (i.e., forces and torques) for the vehicle body is obtained from the presented frequency-limited adaptive control approach and the desired joint velocities for the manipulator are obtained from solving differential kinematics of the UVMS.

The remainder of this paper is organized as follows. It first presents the problem formulation and then introduces the frequency-limited adaptive control for the vehicle body and the kinematic control for the manipulator in Section III. After that, Section IV shows the stability analysis of the vehicle body and the sufficient condition for designing parameters in the control method. The numerical simulations and results are summarized in Section $\mathrm{V}$, followed by conclusions in Section VI.

\section{Problem Formulation}

The control problem of the UVMS consists of two parts: 1) trajectory planning for the vehicle body and the endeffector; and 2) motion control for the vehicle body and the manipulator. This paper focuses on Part 2 given the reference trajectories of the vehicle body and the end-effector. Section III briefly presents a method to generate both trajectories.

Adopting notations from [19], the vehicle body of the UVMS is fully characterized by the position and attitude of the vehicle-fixed frame (denoted as $\mathcal{B}$ ) with respect to (w.r.t.) the inertial frame (denoted as $\mathcal{I}$ ), as shown in Fig. 1. The body position in $\mathcal{I}$ is identified by its Cartesian coordinates denoted as $\boldsymbol{\eta}_{1}=\left[\begin{array}{lll}x & y & z\end{array}\right]^{T} \in \mathbb{R}^{3}$ and the body orientation in $\mathcal{I}$ is described by its Euler angels denoted as $\boldsymbol{\eta}_{2}=[\phi \theta \psi]^{T}$. Then the full configuration of the vehicle body is described by the vector $\boldsymbol{\eta} \in \mathbb{R}^{6}$ defined as $\boldsymbol{\eta}=\left[\boldsymbol{\eta}_{1}^{T} \boldsymbol{\eta}_{2}^{T}\right]^{T}$. The attitude $\boldsymbol{\eta}_{2}$ can be alternatively represented by a rotational matrix denoted as $\boldsymbol{R}_{I}^{B} \in S O(3)$, transforming from $\mathcal{I}$ to $\mathcal{B}$.

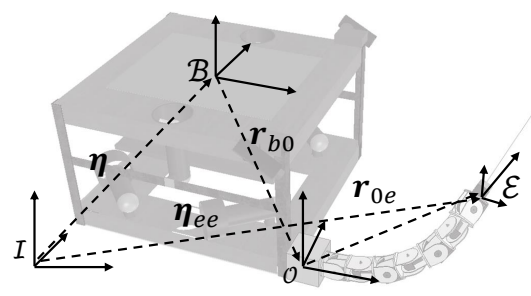

Fig. 1: Reference frames for a UVMS.

Define $\boldsymbol{\nu}_{1} \in \mathbb{R}^{3}\left(\boldsymbol{\nu}_{2} \in \mathbb{R}^{3}\right)$ as the linear (angular) velocity of $\mathcal{B}$ w.r.t. $\mathcal{I}$ expressed in $\mathcal{B}$ and define $\boldsymbol{\nu}=$ $\left[\begin{array}{ll}\boldsymbol{\nu}_{1}^{T} & \boldsymbol{\nu}_{2}^{T}\end{array}\right]^{T}$ as the generalized velocity of the vehicle body. Let $\boldsymbol{q}=\left[q_{1} \cdots q_{n}\right]^{T} \in \mathbb{R}^{n}$ be the vector of manipulator joint positions, where $n$ is the number of joints. Then, the generalized velocity of the UVMS can be written as
$\boldsymbol{\zeta}=\left[\begin{array}{lll}\boldsymbol{\nu}_{1}^{T} & \boldsymbol{\nu}_{2}^{T} & \dot{\boldsymbol{q}}^{T}\end{array}\right]^{T}$, where the operator $\left({ }^{*}\right)$ represents time derivative throughout this paper.

Despite the lack of physical interpretation for $\dot{\boldsymbol{\eta}}_{2}$, the generalized velocity relates to $\dot{\boldsymbol{\eta}}_{1}, \dot{\boldsymbol{\eta}}_{2}$, and $\dot{\boldsymbol{q}}$ through

$$
\boldsymbol{\zeta}=\boldsymbol{J}_{k}\left(\boldsymbol{\eta}_{2}\right)\left[\begin{array}{lll}
\dot{\boldsymbol{\eta}}_{1}^{T} & \dot{\boldsymbol{\eta}}_{2}{ }^{T} & \dot{\boldsymbol{q}}^{T}
\end{array}\right]^{T}
$$

where

$$
\boldsymbol{J}_{k}\left(\boldsymbol{\eta}_{2}\right)=\left[\begin{array}{ccc}
\boldsymbol{R}_{I}^{B} & \boldsymbol{O}_{3 \times 3} & \boldsymbol{O}_{3 \times n} \\
\boldsymbol{O}_{3 \times 3} & \boldsymbol{T}\left(\boldsymbol{\eta}_{\mathbf{2}}\right) & \boldsymbol{O}_{3 \times n} \\
\boldsymbol{O}_{n \times 3} & \boldsymbol{O}_{n \times 3} & \boldsymbol{I}_{n \times n}
\end{array}\right],
$$

the subscript in the form of $n_{1} \times n_{2}$ denotes the matrix dimensionality of $n_{1}$ by $n_{2}, \boldsymbol{I}(\boldsymbol{O})$ denotes an identity (null) matrix, and

$$
\boldsymbol{T}\left(\boldsymbol{\eta}_{2}\right)=\left[\begin{array}{ccc}
1 & 0 & -\cos (\theta) \\
0 & \cos (\phi) & \cos (\theta) \sin (\phi) \\
0 & -\sin (\phi) & \cos (\theta) \sin (\phi)
\end{array}\right] .
$$

The full configuration of the end-effector in the inertial frame $\mathcal{I}$ is defined as the vector $\boldsymbol{\eta}_{e e} \in \mathbb{R}^{6}$ as $\boldsymbol{\eta}_{e e}=$ $\left[\boldsymbol{\eta}_{e e 1}^{T} \boldsymbol{\eta}_{e e 2}^{T}\right]^{T}$, where $\boldsymbol{\eta}_{e e 1} \in \mathbb{R}^{3}$ and $\boldsymbol{\eta}_{e e 2} \in \mathbb{R}^{3}$ denote the position and the attitude of the end-effector, respectively.

The control of the manipulator relies on the differential kinematic chain from the end-effector to the vehicle body. The end-effector velocity in $\mathcal{I}$ is expressed as

$$
\dot{\boldsymbol{\eta}}_{e e}=\boldsymbol{J}\left(\boldsymbol{\eta}_{2}, \boldsymbol{q}\right) \boldsymbol{\zeta}=\left[\begin{array}{l}
\boldsymbol{J}_{1} \\
\boldsymbol{J}_{2}
\end{array}\right] \boldsymbol{\zeta}
$$

where $\boldsymbol{J}_{1}$ and $\boldsymbol{J}_{2}$ are the positional and orientational Jacobian of the end-effector w.r.t. the generalized velocity $\boldsymbol{\zeta}$, and they are given as follows [19],

$$
\boldsymbol{J}_{1}=\left[\begin{array}{lll}
\boldsymbol{R}_{B}^{I} & -S\left(\boldsymbol{r}_{b 0}+\boldsymbol{r}_{0 e}\right) \boldsymbol{R}_{B}^{I} & \boldsymbol{R}_{0}^{I} \boldsymbol{J}_{p}(\boldsymbol{q})
\end{array}\right],
$$

where $\boldsymbol{r}_{b 0}$ and $\boldsymbol{r}_{0 e}$ are vectors in the frame $\mathcal{I}$, as shown in Fig. $1, \boldsymbol{J}_{p}(\boldsymbol{q})$ is the positional geometric Jacobian of the end-effector w.r.t. joint velocities in the frame $\mathcal{O}$ and it can be obtained from Denavit-Hartenberg convention [22]. The rotational matrix from the frame $\mathcal{O}(\mathcal{B})$ to the frame $\mathcal{I}$ is denoted by $\boldsymbol{R}_{0}^{I}\left(\boldsymbol{R}_{B}^{I}\right)$. The operator $\boldsymbol{S}(\cdot)$ is a skew symmetric matrix performing the cross product between two $3 \times 1$ vectors and is given as

$$
\boldsymbol{S}\left(\left[\begin{array}{l}
x_{1} \\
x_{2} \\
x_{3}
\end{array}\right]\right)=\left[\begin{array}{ccc}
0 & -x_{3} & x_{2} \\
x_{3} & 0 & -x_{1} \\
-x_{2} & x_{1} & 0
\end{array}\right]
$$

where $x_{1} \in \mathbb{R}, x_{2} \in \mathbb{R}$, and $x_{3} \in \mathbb{R}$.

The angular velocity of the end-effector is given as

$$
\dot{\boldsymbol{\eta}}_{e e 2}=\boldsymbol{R}_{B}^{I} \boldsymbol{\nu}_{2}+\boldsymbol{R}_{0}^{I} \boldsymbol{J}_{o}(\boldsymbol{q}) \dot{\boldsymbol{q}}
$$

where $\boldsymbol{J}_{o}(\boldsymbol{q})$ is the orientational geometric Jacobian of the end-effector w.r.t. joint velocities in the frame $\mathcal{O}$. Then, the orientational Jacobian is given as

$$
\boldsymbol{J}_{2}=\left[\begin{array}{lll}
\boldsymbol{O}_{3 \times 3} & \boldsymbol{R}_{B}^{I} & \boldsymbol{R}_{0}^{I} \boldsymbol{J}_{o}(\boldsymbol{q})
\end{array}\right] .
$$

In this paper, the dynamical model of the vehicle body is given as [19],

$$
M \dot{\nu}+C(\nu) \nu+D(\nu) \nu+G\left(\eta_{2}\right)=\tau+\tau_{c},
$$


with the following assumption.

Assumptiom 1. The interacting forces between the vehicle body and the manipulator is negligible in comparison with the driving forces from the body thrusters and the hydrodynamic effects.

In Eq. (9), $M \in \mathbb{R}^{6 \times 6}$ denotes the inertial matrix including the added mass from hydrodynamic effects, $C \in \mathbb{R}^{6 \times 6}$ denotes the Coriolis and centripetal terms including the added mass, $\boldsymbol{D} \in \mathbb{R}^{6 \times 6}$ is the friction and hydrodynamic damping effects, and $G \in \mathbb{R}^{6}$ is the vector of gravitational and buoyant generalized forces. All these matrices are unknown. The term $\tau_{c}$ denotes the remaining unmodeled hydrodynamic effects. The generalized driving force, denoted as $\tau$, is produced by the thrusters and can be approximated through the linear mapping [17],

$$
\boldsymbol{\tau}=\boldsymbol{B u},
$$

where $\boldsymbol{B} \in \mathbb{R}^{6 \times p}$ and $\boldsymbol{u} \in \mathbb{R}^{p}$ is the thruster force output.

Assumptiom 2. The matrix $\boldsymbol{B}$ is known and has full rank.

Assumptiom 3. The UVMS state, i.e., $\boldsymbol{\eta}, \boldsymbol{q}$, and $\boldsymbol{\eta}_{e e}$, are observable without error.

With Assumption 2, $\boldsymbol{u}$ can always be found given any $\boldsymbol{\tau}$. Therefore, $\tau$ is referred to as the control input to the UVMS and will be given by the method introduced in Section III. The simulation results in Section $\mathrm{V}$ show that the presented method performs well when observations are corrupted with some additive Gaussian noise.

Problem 1. Given $t_{0} \leq t \leq t_{f}$, find $\boldsymbol{\tau}(t)$ for the system in (9) to track the vehicle reference trajectory denoted as $\boldsymbol{\eta}^{r}(t)$ and find $\dot{\boldsymbol{q}}_{d}(t)$ for the manipulator to track the end-effector reference trajectory denoted as $\boldsymbol{\eta}_{e e}^{r}(t)$, under Assumptions 1 , 2 , and 3 .

\section{Methodology}

This section presents the frequency-limited adaptive control for the vehicle body and the standard kinematic control for the manipulator, given the reference trajectories of the vehicle body and the end-effector. Generation of both reference trajectories is beyond the scope of this paper and more details can be found in [23]. Generally, the end-effector reference can be obtained from planners such as potential fields [24] and random trees [25]. In this paper, the reference trajectory of the vehicle body $\boldsymbol{\eta}^{r}$ is generated from the end-effector reference $\boldsymbol{\eta}_{e e}^{r}$ through two steps. The first step is to shift $\boldsymbol{\eta}_{e e}^{r}$ by a constant vector that is given based on the preferred region [26]. The vehicle body does not need to move much if the end-effector is close to the center of the preferred region. The preferred region functions as a low-pass filter. Then in the second step, the shifted trajectory is smoothed through a filter, e.g., the additive smoothing method [27]. The reference trajectory of the vehicle body could be generated or updated online if necessary.

\section{A. Frequency-limited Adaptive Control}

The frequency-limited adaptive control approach, same as the standard adaptive control, requires an approximation model that can characterize the unknown dynamics modeled in Eq. (9). Exploiting the property of linearity in the parameters [17], Eq. (9) can be rewritten as

$$
\boldsymbol{\Phi}\left(\boldsymbol{R}_{I}^{B}, \boldsymbol{\nu}, \dot{\nu}\right) \boldsymbol{\theta}=\boldsymbol{\tau}
$$

where $\boldsymbol{\theta} \in \mathbb{R}^{m}$ is the parameter vector of the body dynamical model. In this paper, according to [17], $m=26$ and the regressor can be written as

$$
\boldsymbol{\Phi}=\left[\begin{array}{lll}
\boldsymbol{\Phi}_{m} & \boldsymbol{\Phi}_{b g} & \boldsymbol{\Phi}_{c}
\end{array}\right]^{T},
$$

where the matrix block for the buoyancy and gravity forces $\boldsymbol{G}\left(\boldsymbol{\eta}_{2}\right)$ and the block for $\boldsymbol{\tau}_{c}$ are respectively given as

$$
\boldsymbol{\Phi}_{b g}=\left[\begin{array}{cc}
\boldsymbol{R}_{I}^{B} \boldsymbol{z} & \boldsymbol{O}_{3 \times 3} \\
\boldsymbol{O}_{3 \times 1} & \boldsymbol{S}\left(\boldsymbol{R}_{I}^{B} \boldsymbol{z}\right)
\end{array}\right] \text { and } \boldsymbol{\Phi}_{c}=\left[\begin{array}{cc}
\boldsymbol{R}_{I}^{B} & \boldsymbol{O}_{3 \times 3} \\
\boldsymbol{O}_{3 \times 3} & \boldsymbol{R}_{I}^{B}
\end{array}\right]
$$

where $z=[\sin \theta-\cos \theta \sin \phi-\cos \theta \cos \phi]^{T}$. By rewriting $\boldsymbol{\nu}$ as $\left[\begin{array}{llll}u & w & p & q\end{array}\right]^{T}$, the first block $\boldsymbol{\Phi}_{m}$ is given in Eq. (17) at the bottom of this page.

The estimation of $\boldsymbol{\theta}$, denoted as $\hat{\boldsymbol{\theta}}$, is incrementally updated based on the modeling error presented by the mismatch between the actual system state and the desired system state. In the standard adaptive control [13], the modeling parameters are updated as follows,

$$
\dot{\hat{\boldsymbol{\theta}}}=\boldsymbol{K}_{\theta}^{-1} \boldsymbol{\Phi}^{T}\left(\boldsymbol{R}_{I}^{B}, \boldsymbol{\nu}, \dot{\boldsymbol{\nu}}\right) \boldsymbol{s}
$$

where $\boldsymbol{K}_{\theta} \in \mathbb{R}^{6 \times 6}$ is positive definite. The mismatch is

$$
\boldsymbol{s}=\left[\begin{array}{cc}
\boldsymbol{R}_{I}^{B} & \boldsymbol{O}_{3 \times 3} \\
\boldsymbol{O}_{3 \times 3} & \boldsymbol{T}\left(\boldsymbol{\eta}_{\mathbf{2}}\right)
\end{array}\right]\left(\dot{\boldsymbol{\eta}}^{r}-\dot{\boldsymbol{\eta}}\right)+\boldsymbol{\Lambda}\left[\begin{array}{c}
\boldsymbol{R}_{I}^{B}\left(\boldsymbol{\eta}_{1}^{r}-\boldsymbol{\eta}_{1}\right) \\
\tilde{\varepsilon}
\end{array}\right],
$$

where $\boldsymbol{\Lambda}$ is positive definite and $\{\tilde{\eta}, \tilde{\varepsilon}\}$ is the error of the body attitude in the quaternion format [19]. The rule of updating $\hat{\boldsymbol{\theta}}$ in frequency-limited adaptive control will be presented after the introduction of the control law.

Same as the standard one, the control law in frequencylimited adaptive control is given by

$$
\boldsymbol{\tau}=\boldsymbol{K}_{D} \boldsymbol{s}^{\prime}+\boldsymbol{\Phi}\left(\boldsymbol{R}_{I}^{B}, \boldsymbol{\nu}, \dot{\boldsymbol{\nu}}\right) \hat{\boldsymbol{\theta}}
$$

$$
\boldsymbol{\Phi}_{m}=\left[\begin{array}{ccccccccccccccccccccccccc}
\dot{u}+q w-r v & -q^{2}-r^{2} & p q-\dot{p} & \dot{q}+p r & 0 & 0 & 0 & 0 & 0 & 0 & -\dot{u} & r v-q w & 0 & 0 & 0 \\
\dot{v}-p w+r u & \dot{p}+p q & -p^{2}-r^{2} & q r-\dot{p} & 0 & 0 & 0 & 0 & 0 & 0 & -r u & -\dot{v} & p w & 0 & 0 & 0 \\
\dot{w}+p v-q u & p r-\dot{q} & \dot{p}+q r & -p^{2}-q^{2} & 0 & 0 & 0 & 0 & 0 & 0 & q u & -p v & -\dot{w} & 0 & 0 & 0 \\
0 & 0 & \dot{w}+p v-q u & p w-\dot{v}-r u & \dot{p} & -q r & q r & p r-\dot{q} & r^{2}-q^{2}-\dot{p}-p q & 0 & v w-v w & -\dot{p} & q r & -q r \\
0 & q u-p v-\dot{w} & 0 & \dot{u}+q w-r v & p r & \dot{q} & -p r-\dot{p}-q r & p q-\dot{p} & p^{2}-r^{2}-u w & 0 & u w-p r & -\dot{q} & p r \\
0 & \dot{v}-p w+r u & r v-q w-\dot{u} & 0 & -p q & p q & \dot{p} & q^{2}-p^{2}-\dot{q}-p r & q r-\dot{p} & u v & -u v & 0 & p q-p q-\dot{p}
\end{array}\right]
$$


where

$$
\begin{aligned}
\boldsymbol{s}^{\prime} & =\left[\begin{array}{cc}
\boldsymbol{R}_{I}^{B} & \boldsymbol{O}_{3 \times 3} \\
\boldsymbol{O}_{3 \times 3} & \boldsymbol{T}\left(\boldsymbol{\eta}_{2}\right)
\end{array}\right]\left(\dot{\boldsymbol{\eta}}^{r}-\dot{\boldsymbol{\eta}}\right) \\
& +\left\{\boldsymbol{\Lambda}+\left[\begin{array}{cc}
k_{p} \boldsymbol{I}_{3 \times 3} & \boldsymbol{O}_{3 \times 3} \\
\boldsymbol{O}_{3 \times 3} & k_{o} \boldsymbol{I}_{3 \times 3}
\end{array}\right]\right\}\left[\begin{array}{c}
\boldsymbol{R}_{I}^{B}\left(\boldsymbol{\eta}_{1}^{r}-\boldsymbol{\eta}_{1}\right) \\
\tilde{\boldsymbol{\varepsilon}}
\end{array}\right] .
\end{aligned}
$$

It can be seen that the mismatch $s$ is directly injected into the parameter updating law (14). Therefore the mismatch can introduce oscillations of high frequency in $\hat{\boldsymbol{\theta}}$ when it is large at transient instants. Then the high-frequency oscillations in $\hat{\boldsymbol{\theta}}$ result in undesired oscillations in control inputs through (16) and thus in the state of the vehicle body.

In order to prohibit undesired oscillations, similar to [20], an auxiliary model is introduced to regulate the learning process of the model parameters in this paper. Using the same regressor in Eq. (11), this auxiliary model is given as

$$
\boldsymbol{\Phi}\left(\boldsymbol{R}_{I}^{B}, \boldsymbol{\nu}, \dot{\boldsymbol{\nu}}\right) \boldsymbol{\theta}_{f}=\boldsymbol{\tau} .
$$

The learning rule of its parameter estimation $\hat{\boldsymbol{\theta}}_{f}$ is driven by the mismatch between the approximation model and the auxiliary model as follows

$$
\dot{\hat{\boldsymbol{\theta}}}_{f}=\boldsymbol{K}_{f}\left(\hat{\boldsymbol{\theta}}-\hat{\boldsymbol{\theta}}_{f}\right),
$$

where $\boldsymbol{K}_{f} \in \mathbb{R}^{6 \times 6}$ is the positive definite filter gain.

Then, different from the standard method (14), the updating rule for $\hat{\boldsymbol{\theta}}$ in frequency-limited adaptive control is counteracted by the mismatch as follows,

$$
\dot{\hat{\boldsymbol{\theta}}}=\boldsymbol{K}_{\theta}^{-1} \boldsymbol{\Phi}^{T}\left(\boldsymbol{R}_{I}^{B}, \boldsymbol{\nu}, \dot{\boldsymbol{\nu}}\right) \boldsymbol{s}-\boldsymbol{K}_{c}\left(\hat{\boldsymbol{\theta}}-\hat{\boldsymbol{\theta}}_{f}\right),
$$

where $\boldsymbol{K}_{c}$ is a positive definite matrix. Equations (11), (16), (20), and (21) together compose the frequency-limited adaptive control approach for the UVMS vehicle body.

\section{B. Kinematic Control of Manipulator}

An UVMS is kinematically redundant since it possesses more degree of freedoms than those required to track the endeffector reference trajectory. Kinematic control based on null space of the end-effector Jacobian matrix allows exploiting such redundancy to increase manipulability. The kinematic control presented here is standard and can be found in [19].

The primary task is defined as the end-effector reference tracking, while the second task is designed to increase manipulability [28], which is defined as the determinant of the Jacobian matrix block w.r.t $\dot{\boldsymbol{q}}$ (denoted as $\boldsymbol{J}_{q}$ ) according to Eq. (4). Then, by using Jacobian inverse techniques based on the task priorities, the desired joint velocities for the manipulator are given as

$$
\begin{aligned}
\dot{\boldsymbol{q}}_{d}= & \boldsymbol{J}_{f}^{\dagger}\left(\tilde{\boldsymbol{v}}_{e e 1}+\boldsymbol{\Lambda}_{1} \tilde{\boldsymbol{\eta}}_{e e 1}\right) \\
& +\left[\boldsymbol{J}_{s}\left(\boldsymbol{I}-\boldsymbol{J}_{f}^{\dagger} \boldsymbol{J}_{f}\right)\right]^{\dagger}\left[\frac{\partial\left|\boldsymbol{J}_{q}\right|}{\partial \boldsymbol{q}}-\boldsymbol{J}_{s} \boldsymbol{J}_{f}^{\dagger}\left(\tilde{\boldsymbol{v}}_{e e 1}+\boldsymbol{\Lambda}_{1} \tilde{\boldsymbol{\eta}}_{e e 1}\right)\right],
\end{aligned}
$$

where $\boldsymbol{\Lambda}_{1}$ is positive definite, the superscript $\dagger$ denotes the Moore-Penrose pseudoinverse [29], $|\cdot|$ is the determinant operator, and $\boldsymbol{J}_{f}$ and $\boldsymbol{J}_{s}$ are the $\boldsymbol{q}$ dependent primary and secondary task Jacobians from Eq. (4), respectively. The velocity and position errors in the frame $\mathcal{I}$ is respectively given by

$$
\tilde{\boldsymbol{v}}_{e e 1}=\dot{\boldsymbol{\eta}}_{e e 1}^{r}-\boldsymbol{J}\left[\dot{\boldsymbol{\eta}}_{1} \dot{\boldsymbol{\eta}}_{2} \boldsymbol{O}\right]^{T}, \tilde{\boldsymbol{\eta}}_{e e 1}=\boldsymbol{\eta}_{e e 1}^{r}-\boldsymbol{\eta}_{e e 1} .
$$

\section{Stability Analysis}

In paper [30], it has been proved that 1) the inertia matrix of the vehicle body system is positive definite and symmetric; 2) the damping matrix is positive definite; and 3) the matrix $\dot{M}-2 \boldsymbol{C}(\boldsymbol{\nu})$ is skew symmetric, i.e., $\boldsymbol{\nu}^{T}[\dot{\boldsymbol{M}}-2 \boldsymbol{C}(\boldsymbol{q}, \boldsymbol{\nu})] \boldsymbol{\nu}=$ $\boldsymbol{O}, \forall \boldsymbol{\nu} \in \mathbb{R}^{6}$. Then we have the following theorem.

Theorem 1. The closed-loop dynamical system of the vehicle body, defined over the approximation model given by Eq. (11), the auxiliary model given by Eq. (19), the feedback control law given by Eq. (16) and the learning rules given by Eq. (21) and Eq. (20), is asymptotically stable between transient instants.

Proof. Consider the following Lyapunov function candidate

$$
\begin{aligned}
\mathcal{V}= & \frac{1}{2} \boldsymbol{s}^{T} \boldsymbol{M} \boldsymbol{s}+\frac{1}{2}(\boldsymbol{\theta}-\hat{\boldsymbol{\theta}})^{T} \boldsymbol{K}_{\theta}(\boldsymbol{\theta}-\hat{\boldsymbol{\theta}})+\frac{1}{2}\left(\boldsymbol{\theta}-\hat{\boldsymbol{\theta}}_{f}\right)^{T} \boldsymbol{K}\left(\boldsymbol{\theta}-\hat{\boldsymbol{\theta}}_{f}\right) \\
& +\frac{1}{2}\left[\begin{array}{c}
\boldsymbol{\eta}_{1}^{r}-\boldsymbol{\eta}_{1} \\
\tilde{\boldsymbol{z}}
\end{array}\right]^{T}\left[\begin{array}{cc}
k_{p} \boldsymbol{I}_{3} & \boldsymbol{O}_{3 \times 4} \\
\boldsymbol{O}_{4 \times 3} & 2 k_{o} \boldsymbol{I}_{4}
\end{array}\right]\left[\begin{array}{c}
\boldsymbol{\eta}_{1}^{r}-\boldsymbol{\eta}_{1} \\
\tilde{\boldsymbol{z}}
\end{array}\right],
\end{aligned}
$$

where $\tilde{\boldsymbol{z}}=\left[\begin{array}{ll}1 & \mathbf{0}^{T}\end{array}\right]^{T}-\boldsymbol{z}=\left[1-\tilde{\eta}-\tilde{\boldsymbol{\varepsilon}}^{T}\right], k_{o}$ and $k_{p}$ are positive.

It is quite obvious that $\mathcal{V}$ is semi-positive definite. Differentiating $\mathcal{V}$ with respect to time yields

$$
\begin{aligned}
\dot{\mathcal{V}}= & \frac{1}{2} \boldsymbol{s}^{T} \dot{\boldsymbol{M}} \boldsymbol{s}+\boldsymbol{s}^{T} \boldsymbol{M} \dot{\boldsymbol{s}}+(\boldsymbol{\theta}-\hat{\boldsymbol{\theta}})^{T} \boldsymbol{K}_{\theta}(\dot{\boldsymbol{\theta}}-\dot{\hat{\boldsymbol{\theta}}}) \\
& +\left(\boldsymbol{\theta}-\hat{\boldsymbol{\theta}}_{f}\right)^{T} \boldsymbol{K}\left(\dot{\boldsymbol{\theta}}-\dot{\hat{\boldsymbol{\theta}}}_{f}\right)+k_{p}\left(\boldsymbol{\eta}_{1}^{r}-\boldsymbol{\eta}_{1}\right)^{T} \boldsymbol{R}_{B}^{I}\left(\boldsymbol{\nu}_{1}^{r}-\boldsymbol{\nu}_{1}\right) \\
& -k_{o} \tilde{\boldsymbol{z}}^{T}\left[\begin{array}{c}
\tilde{\eta} \boldsymbol{I}_{3 \times 3}+\boldsymbol{S}(\tilde{\boldsymbol{\varepsilon}}) \\
\tilde{\boldsymbol{\varepsilon}}^{T}
\end{array}\right]\left(\boldsymbol{\nu}_{2}^{r}-\boldsymbol{\nu}_{2}\right) .
\end{aligned}
$$

Rewrite the term $M \dot{s}$ in above equation as

$$
\boldsymbol{M} \dot{\boldsymbol{s}}=\boldsymbol{M} \dot{\boldsymbol{\nu}}^{d}-\boldsymbol{\tau}+\boldsymbol{C}(\boldsymbol{\nu}) \boldsymbol{\nu}+\boldsymbol{D}(\boldsymbol{\nu}) \boldsymbol{\nu}+\boldsymbol{G},
$$

where $\boldsymbol{\nu}^{d}=\boldsymbol{\nu}^{r}+\boldsymbol{\Lambda}\left[\begin{array}{c}\boldsymbol{R}_{I}^{B}\left(\boldsymbol{\eta}_{1}^{r}-\boldsymbol{\eta}_{1}\right) \\ \tilde{\varepsilon}\end{array}\right]$.

Substituting Eq. (26) into Eq. (25) yields

$$
\begin{aligned}
\dot{\mathcal{V}}= & -\boldsymbol{s}^{T} \boldsymbol{D} \boldsymbol{s}-(\boldsymbol{\theta}-\hat{\boldsymbol{\theta}})^{T} \boldsymbol{K}_{\theta} \dot{\hat{\boldsymbol{\theta}}}-\left(\boldsymbol{\theta}-\hat{\boldsymbol{\theta}}_{f}\right)^{T} \boldsymbol{K} \dot{\hat{\boldsymbol{\theta}}}_{f} \\
& +\boldsymbol{s}^{T}\left[\boldsymbol{M} \dot{\boldsymbol{\nu}}^{d}-\boldsymbol{\tau}+\boldsymbol{C}(\boldsymbol{\nu}) \boldsymbol{\nu}^{d}+\boldsymbol{D}(\boldsymbol{\nu}) \boldsymbol{\nu}^{d}+\boldsymbol{G}\right] \\
& +\boldsymbol{s}^{T}\left[\begin{array}{c}
k_{p} \boldsymbol{R}_{B}^{I}\left(\boldsymbol{\eta}_{1}^{r}-\boldsymbol{\eta}_{1}\right) \\
k_{0} \tilde{\varepsilon}
\end{array}\right] \\
& -k_{p} \lambda_{p}\left(\boldsymbol{\eta}_{1}^{r}-\boldsymbol{\eta}_{1}\right)^{T}\left(\boldsymbol{\eta}_{1}^{r}-\boldsymbol{\eta}_{1}\right)-\lambda_{0} k_{0} \tilde{\varepsilon}^{T} \tilde{\varepsilon},
\end{aligned}
$$

where the actual parameter vector $\boldsymbol{\theta}$ between transient instants is constant or slowly changing, i.e, $\dot{\boldsymbol{\theta}}=0$.

After that, substituting parameter updating laws (20) and (21) and the control rule (16) into the above equation yields

$$
\begin{aligned}
\dot{\mathcal{V}}= & -\boldsymbol{s}^{T} \boldsymbol{D}(\boldsymbol{\nu}) \boldsymbol{s}-(\boldsymbol{\theta}-\hat{\boldsymbol{\theta}})^{T} \boldsymbol{K}_{\theta} \dot{\hat{\boldsymbol{\theta}}} \\
= & -k_{p} \lambda_{p}\left(\boldsymbol{\eta}_{1}^{r}-\boldsymbol{\eta}_{1}\right)^{T}\left(\boldsymbol{\eta}_{1}^{r}-\boldsymbol{\eta}_{1}\right)-\lambda_{0} k_{0} \tilde{\varepsilon}^{T} \tilde{\varepsilon} \\
& -\boldsymbol{s}^{T}\left(\boldsymbol{D}(\boldsymbol{\nu})+\boldsymbol{K}_{D}\right) \boldsymbol{s} \\
& +(\boldsymbol{\theta}-\hat{\boldsymbol{\theta}})^{T}\left(\boldsymbol{K}_{c} \boldsymbol{K}_{\theta}-\boldsymbol{K} \boldsymbol{K}_{f}\right)\left(\hat{\boldsymbol{\theta}}-\hat{\boldsymbol{\theta}}_{f}\right) \\
& -\left(\hat{\boldsymbol{\theta}}-\hat{\boldsymbol{\theta}}_{f}\right)^{T} \boldsymbol{K} \boldsymbol{K}_{f}\left(\hat{\boldsymbol{\theta}}-\hat{\boldsymbol{\theta}}_{f}\right) .
\end{aligned}
$$


From the above equation, it can be observed that a sufficient condition for stabilizing the controlled robotic system is the existence of a positive definite matrix $\boldsymbol{K}$ such that $\boldsymbol{K}_{f} \boldsymbol{K}=\boldsymbol{K}_{c} \boldsymbol{K}_{\theta}$. Then the time derivative of the Lyapunov candidate can be written as

$$
\begin{aligned}
\dot{\mathcal{V}}= & -k_{p} \lambda_{p}\left(\boldsymbol{\eta}_{1}^{r}-\boldsymbol{\eta}_{1}\right)^{T}\left(\boldsymbol{\eta}_{1}^{r}-\boldsymbol{\eta}_{1}\right)-\lambda_{0} k_{0} \tilde{\varepsilon}^{T} \tilde{\boldsymbol{\varepsilon}} \\
& -\boldsymbol{s}^{T}\left\{\boldsymbol{D}(\boldsymbol{\nu})+\left[\begin{array}{cc}
k_{p} \boldsymbol{I}_{3 \times 3} & \boldsymbol{O}_{3 \times 3} \\
\boldsymbol{O}_{3 \times 3} & k_{o} \boldsymbol{I}_{3 \times 3}
\end{array}\right]\right\} \boldsymbol{s} \\
& -\left(\hat{\boldsymbol{\theta}}-\hat{\boldsymbol{\theta}}_{f}\right)^{T} \boldsymbol{K}_{f} \boldsymbol{K}\left(\hat{\boldsymbol{\theta}}-\hat{\boldsymbol{\theta}}_{f}\right) .
\end{aligned}
$$

Thus $\dot{\mathcal{V}} \leq 0$ and the system can then be proved stable using the Barbalat's Lemma. Since $\mathcal{V}$ is semi-positive definite, $\dot{\mathcal{V}} \leq$ 0 , and $\dot{\mathcal{V}}$ is uniformly continuous, then $\tilde{\varepsilon} \rightarrow 0, s \rightarrow 0$, and $\boldsymbol{\eta}_{1} \rightarrow \boldsymbol{\eta}_{1}^{r}$ as $t \rightarrow \infty$.

\section{Simulation Results}

The effectiveness of the presented frequency-limited adaptive control is demonstrated by the performance comparisons with the standard adaptive control through numerical examples simulated in V-rep [31]. V-rep is a virtual robot experimentation platform that simulates interactions between rigid bodies. The scene built in V-rep involves a UVMS equipped with one manipulator of 7 DOFs, as shown in Fig. 2, where the end-effector is a Diving-PAM underwater fluorometer used to take measurements of photosynthesis of underwater plants.

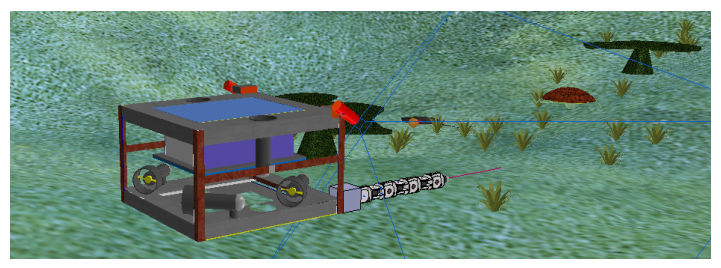

Fig. 2: A simulation scenario built in V-rep.

Note that V-rep does not simulate fluid dynamics, therefore the hydrodynamical forces are imposed to the physics engine in V-rep explicitly and they are given by [17]. For example, the drag force is simulated by

$$
\boldsymbol{f}_{c}=c\left(\boldsymbol{v}_{c}-\boldsymbol{\nu}_{1}\right)\left\|\boldsymbol{v}_{c}-\boldsymbol{\nu}_{1}\right\|_{2},
$$

where the constant $c$ depends on the effective area of the vehicle body and fluid characteristics and is usually obtained through experiments, $\|\cdot\|_{2}$ denotes Euclidean norm, and $\boldsymbol{v}_{c}$ is the fluid velocity in the frame $\mathcal{B}$. The fluid velocity is simulated using the theory of superposition [9]. An example of the hydrodynamical forces is shown in Fig. 3a, where the forces in $\mathrm{y}$ and $\mathrm{z}$ directions and the torques in all directions are designed to be negligible in order to better show the relation between disturbances and processes of learning modelling parameters.

The reference trajectories of the vehicle body (solid curve) and the end-effector (dashed curve) are shown in Fig. 3b, where the body reference trajectory is obtained using the method introduced in Section III. The primary task of the

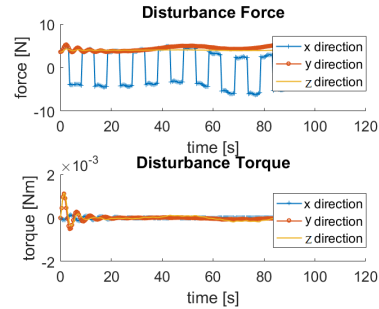

(a)

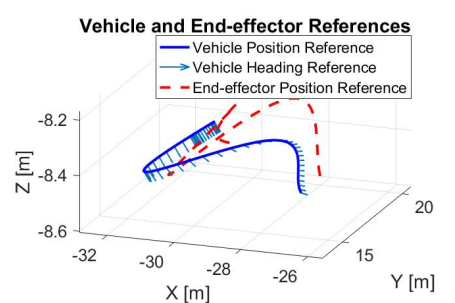

(b)
Fig. 3: (a) Forces and torques from wave disturbances; (b) Reference trajectories.

end-effector is to track the end-effector position trajectory given in the inertial frame $\mathcal{I}$.

The frequency-limited adaptive control and the standard adaptive control were directly applied to the vehicle body. The tracking error of the body from both methods are shown in Fig. $4 a$ and $4 b$, respectively. It can be seen that the frequency-limited method resulted in less tracking error and more stable state at the beginning of the simulation (the initial modeling error was set to be large), thanks to its mechanism of prohibiting high-frequency oscillations. In this case, both methods produced tracking error of similar amplitude after 80 seconds, however the vehicle body controlled by the standard method has oscillations of higher frequency.

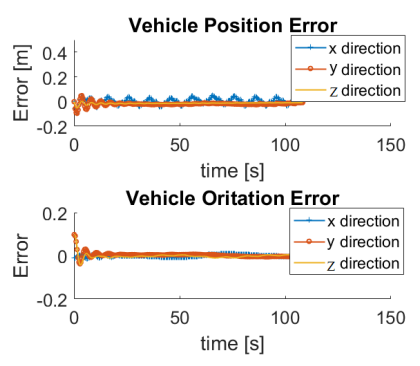

(a)

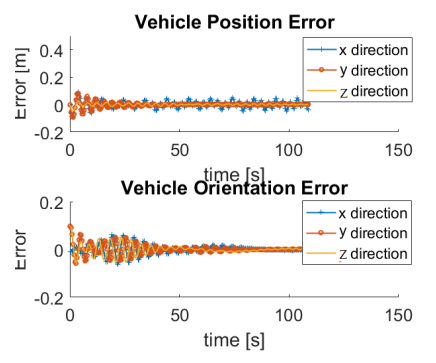

(b)
Fig. 4: Vehicle tracking error. (a) Frequency-limited method; (b) Standard method.

By comparing the end-effector tracking error after 80 seconds in Fig. 5a and Fig. 5b, it is clear that these oscillations of high-frequency has negative effect on the tracking performance of the end-effector. The high-frequency and large amplitude oscillations in the vehicle body require the manipulator to move into its configuration regions that have low manipulability.

These undesired oscillations were produced from the highfrequency oscillations in modeling parameters as shown in Fig. 6a and Fig. 6b. The adaptation processes of three parameters that are closely related to the hydrodynamic forces in $\mathrm{x}$ direction are depicted. The parameters obtained from the frequency-limited adaptive control approach have lower-frequency and smaller-amplitude oscillations, and thus produced better control inputs.

The spectrums of control inputs resulted from the 


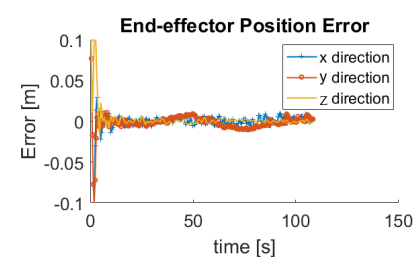

(a)

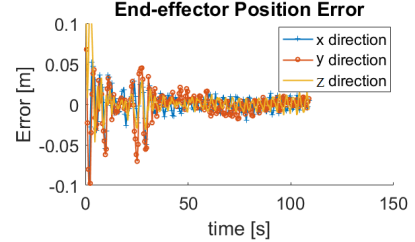

(b)
Fig. 5: End-effector tracking error. (a) Frequency-limited method; (b) Standard method.

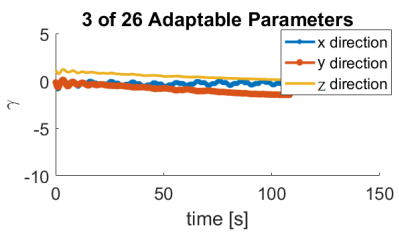

(a)

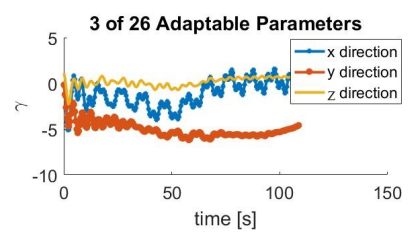

(b)
Fig. 6: Parameter adaptation processes. (a) Frequency-limited method; (b) Standard method.

frequency-limited adaptive control (solid curve) and the standard adaptive control (dashed curve) are illustrated in Fig. 7. This comparison demonstrates that the frequencylimited method can effectively reduce the control signal of high frequency, which is large in control signal produced by the standard method.

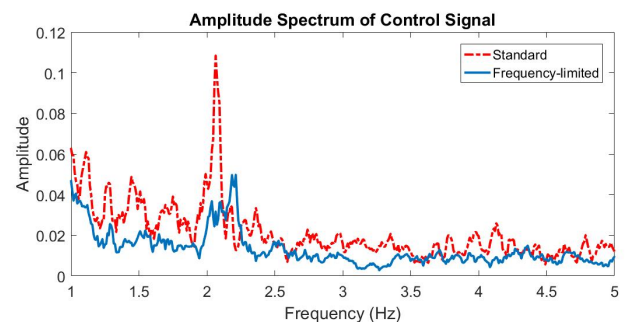

Fig. 7: Amplitude spectrum of control signals.

\section{CONCLUSION}

The frequency-limited adaptive control approach is effective in stabilizing vehicle body for the on-board manipulator to track end-effector reference trajectory. High-frequency oscillations in modeling UVMS dynamics can be reduced by counteracting the mismatch between the approximation model and the auxiliary model. The resultant control input consists of less high-frequency signals than the one generated by the standard adaptive control. A sufficient condition on control parameters is proposed for guaranteed robotic system stability.

\section{REFERENCES}

[1] M. Sasil-Orbita and H. Mukai. A comparative study of the photosynthetic activity among three temperate seagrass species in northern japan. Marine Ecology, 27(4):388-396.
[2] J. Evans, P. Redmond, C. Plakas, K. Hamilton, and D. Lane. Autonomous docking for intervention-auvs using sonar and video-based real-time 3d pose estimation. In OCEANS 2003. Proceedings, volume 4, pages 2201-2210 Vol.4, Sept 2003.

[3] G. Marani, S. Choi, and J. Yuh. Underwater autonomous manipulation for intervention missions $\{$ AUVs $\}$. Ocean Engineering, 36(1):15 - 23, 2009. Autonomous Underwater Vehicles.

[4] P. Sanz, P. Ridao, G. Oliver, C. Melchiorri, G. Casalino, C. Silvestre, Y. Petillot, and A. Turetta. 7th ifac symposium on intelligent autonomous vehicles trident: A framework for autonomous underwater intervention missions with dexterous manipulation capabilities. IFAC Proceedings Volumes, 43(16):187 - 192, 2010.

[5] P. Cieslak, P. Ridao, and M. Giergiel. Autonomous underwater panel operation by girona500 uvms: A practical approach to autonomous underwater manipulation. In 2015 IEEE International Conference on Robotics and Automation (ICRA), pages 529-536, May 2015.

[6] F. Maurelli, M. Carreras, J. Salvi, D. Lane, K. Kyriakopoulos, G. Karras, M. Fox, D. Long, P. Kormushev, and D. Caldwell. The pandora project: A success story in auv autonomy. In OCEANS 2016 Shanghai, pages 1-8, April 2016.

[7] J. Woolfrey, D. Liu, and M. Carmichael. Kinematic control of an autonomous underwater vehicle-manipulator system (auvms) using autoregressive prediction of vehicle motion and model predictive control. In 2016 IEEE International Conference on Robotics and Automation (ICRA), pages 4591-4596, May 2016.

[8] M. Fine, E. Meroz-Fine, and O. Hoegh-Guldberg. Tolerance of endolithic algae to elevated temperature and light in the coral montipora monasteriata from the southern great barrier reef. Journal of Experimental Biology, 208(1):75-81, 2004.

[9] R. Dean and R. Dalrymple. Water Wave Mechanics for Engineers and Scientists. Advanced series on ocean engineering. World Scientific, 1991.

[10] B. Subudhi, K. Mukherjee, and S. Ghosh. A static output feedback control design for path following of autonomous underwater vehicle in vertical plane. Ocean Engineering, 63:72 - 76, 2013.

[11] V. Venkatesan, S. Mohan, and J. Kim. Disturbance observer based terminal sliding mode control of an underwater manipulator. In Control Automation Robotics Vision (ICARCV), 2014 13th International Conference on, pages 1566-1572, Dec 2014.

[12] H. Esfahani, V. Azimirad, and M. Danesh. A time delay controller included terminal sliding mode and fuzzy gain tuning for underwater vehicle-manipulator systems. Ocean Engineering, 107:97 - 107, 2015.

[13] G. Antonelli, F. Caccavale, and S. Chiaverini. Adaptive tracking control of underwater vehicle-manipulator systems based on the virtual decomposition approach. IEEE Transactions on Robotics and Automation, 20(3):594-602, June 2004.

[14] J. Vannoy and J. Xiao. Real-time adaptive motion planning (ramp) of mobile manipulators in dynamic environments with unforeseen changes. IEEE Transactions on Robotics, 24(5):1199-1212, Oct 2008.

[15] M. Galicki. An adaptive non-linear constraint control of mobile manipulators. Mechanism and Machine Theory, 88:63 - 85, 2015.

[16] D. Williams, F. Baralli, M. Micheli, and S. Vasoli. Adaptive underwater sonar surveys in the presence of strong currents. In 2016 IEEE International Conference on Robotics and Automation, May 2016.

[17] T. Fossen. Guidance and control of ocean vehicles. Wiley, 1994.

[18] M. Santhakumar. A nonregressor nonlinear disturbance observer-based adaptive control scheme for an underwater manipulator. Advanced Robotics, 27(16):1273-1283, 2013.

[19] G. Antonelli. Underwater Robots: Motion and Force Control of Vehicle-Manipulator Systems. Springer Tracts in Advanced Robotics. Springer Berlin Heidelberg, 2010.

[20] T. Yucelen, G. De La Torre, and E. Johnson. Frequency-limited adaptive control architecture for transient response improvement. In 2013 American Control Conference, pages 6631-6636, June 2013.

[21] J. Yuh, S. Zhao, and P. Lee. Application of adaptive disturbance observer control to an underwater manipulator. In 2001 IEEE International Conference on Robotics and Automation (ICRA), volume 4, pages 3244-3249 vol.4, 2001.

[22] B. Siciliano, L. Sciavicco, L. Villani, and G. Oriolo. Robotics: modelling, planning and control. Springer Science \& Business Media, 2010.

[23] P. Sotiropoulos, V. Kolonias, N. Aspragathos, and E. Housos. Fast underwater vehicle manipulator system motion planning in gpus. In Control Automation (MED), 2013 21st Mediterranean Conference on, pages 1168-1173, June 2013. 
[24] W. Lu, G. Zhang, and S. Ferrari. An information potential approach to integrated sensor path planning and control. IEEE Transactions on Robotics, in press.

[25] W. Lu, G. Zhang, and S. Ferrari. A randomized hybrid system approach to coordinated robotic sensor planning. In IEEE Conference on Decision and Control, pages 3857-3864, 2010.

[26] Y. Yamamoto and X. Yun. Control of mobile manipulators following a moving surface. In Robotics and Automation, 1993. Proceedings., 1993 IEEE International Conference on, pages 1-6 vol.3, 1993.

[27] C. Manning, P. Raghavan, and H. Schütze. Introduction to Information Retrieval. An Introduction to Information Retrieval. Cambridge University Press, 2008.

[28] T. Yoshikawa. Manipulability of robotic mechanisms. The international journal of Robotics Research, 4(2):3-9, 1985.

[29] A. Ben-Israel and T. Greville. Generalized Inverses: Theory and Applications. CMS Books in Mathematics. Springer, 2003.

[30] I. Schjlberg and T. Fossen. Modelling and control of underwater vehicle-manipulator systems. In in Proc. rd Conf. on Marine Craft maneuvering and control, pages 45-57, 1994.

[31] E. Rohmer, S. Singh, and M. Freese. V-rep: a versatile and scalable robot simulation framework. In Proc. of The International Conference on Intelligent Robots and Systems (IROS), 2013. 